\title{
A multiplex real-time PCR assay for identification of multi-drug
}

\section{resistant Gram-negative pathogens}

María P. Torres', Ph.D., Laura R. Porter', Stacey Morrow², MS, MT(ASCP), Christopher M. Connelly', Ph.D., and

Nancy D. Hanson², Ph.D.

${ }^{1}$ Research and Development Division, Streck Inc., 7002 S. 109th Street, Omaha, NE 68128.

Electronic address: cconnelly@streck.com

${ }^{2}$ Center for Research in Anti-Infectives and Biotechnology, Department of Medical Microbiology and Immunology, Creighton University School of Medicine, 2500 California Plaza, Omaha, NE 68178. Electronic address: ndhanson@ creighton.edu

\section{Introduction}

As part of the global effort to combat antibiotic resistance, several organizations have proposed both the establishment of surveillance networks and implementation of antimicrobial stewardship programs. This has highlighted the need for molecular methods with improved sensitivity over current phenotypic susceptibility tests permitting a reduction in the time needed to obtain results. The interpretation of traditional culture-based assays, based on the observed phenotype, is complex and can fail to detect the presence of some clinically important resistance genes when they are disseminated on mobile genetic elements or co-existing in multi-drug resistant infections. Incomplete identification and spread of these organisms can cause infection control issues that impact hospital infection rates and reduce the effectiveness of antibiotics, potentially increasing mortality rates of infected patients. Therefore, novel diagnostic tests that lead to the accurate identification of these resistance mechanisms are needed to improve and complement current practices for antibiotic stewardship and infection control. To this end, we have developed two different multiplex real-time PCR kits that together target a comprehensive list ( $450 \beta$-lactamase gene variants) of Gram-negative bacterial genotypes conferring resistance to the largest class of clinically available antibiotics, the $\beta$-Lactams. These tests are provided in a convenient multiplex PCR format that

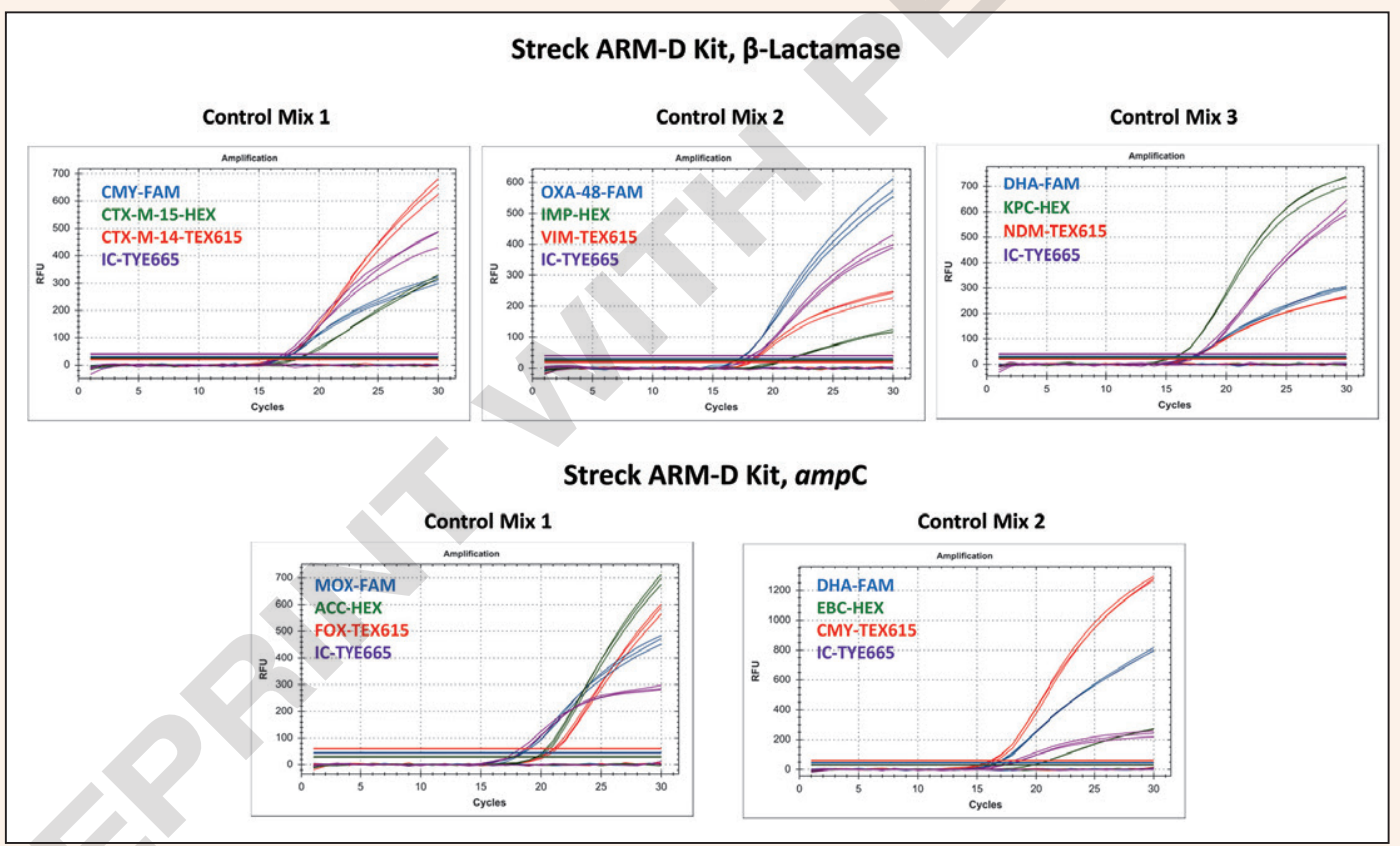

Figure 1. Multiplex amplification of positive DNA Controls $(n=3)$ using Streck ARM-D Kits, $\beta$-Lactamase and Streck ARM-D Kit, ampC. identifies resistance mechanisms encoded by the bacteria present in the samples. The ARM-D ${ }^{\circledR}$ Kit, $\beta$-Lactamase targets 9 of the most prevalent $\beta$-lactamase gene families worldwide; the Streck ARM-D Kit, ampC identifies 6 gene families associated with plasmidmediated mechanisms of antibiotic resistance. Used independently or in combination with the phenotypic assays, the Streck ARM-D Kits can detect and identify the genotypic determinants of resistance in a shorter time and with a higher degree of confidence than current methods alone.

\section{Materials and Methods}

Streck Antibiotic Resistance Monitoring and Detection (ARM-D) Kits, $\beta$-Lactamase and ampC (Streck, Omaha, NE) were used to detect the following $\beta$-lactamase genes: CMY-2, CTX-M-14, CTX-M-15, OXA-48, IMP, VIM, DHA, KPC, and NDM; and plasmid-me- 
diated ampCs: MOX, ACC, FOX, DHA, $\mathrm{EBC}$, and $\mathrm{CMY}-2$. An internal control (IC) that targets a conserved region in the genome of Gram-negative bacteria was included in each mix to identify potential false negative results. The target genes were detected using specific primers and fluorescently-labeled hydrolysis probes. Real-time PCR was carried out on various instruments including the following platforms: Streck Zulu RTTM, Bio-Rad CFX96 Touch'm, ABI 7500 Fast, $A B I$ QuantStudio ${ }^{\circledR} 7$ Flex and QIAGEN $^{\circledR}$ Rotor-Gene $Q$ according to manufacturer's instructions. Reaction conditions were as described in the kits' instructions provided by the manufacturer (see www.streck.com for details on each kit).

DNA was purified from $\beta$-lactamasepositive and -negative overnight bacterial cultures $(n=30)$ that had been previously characterized by singleplex PCR and/or sequencing. DNA was isolated using the QIAGEN DNeasy ${ }^{\circledR}$ Blood and Tissue Kit per the manufacturer's instructions. Controls provided with the kit were used to confirm PCR assay performance and positive gene target identification based on guidelines provided in manufacturer's instructions for the ARM-D Kits. Sensitivity and specificity of the assays were calculated based on the ability to correctly identify the positive and negative clinical isolates, respectively.

\section{Results and Discussion}

The results shown in Figure 1 illustrate the amplification of positive controls for the $\beta$-lactamase gene families that are detected by each multiplex PCR mix within each kit. In this study, the kit was tested with previously characterized clinical isolates ( $\mathrm{n}=30$ ). All isolates included in the study were positive for internal control (IC) included in all the PCR mixes. Ca values for positive controls and $\beta$-lactamase-positive clinical isolates were under 21 cycles for all the targets detected. Out of the 30 isolates tested using the $\beta$-Lactamase Kit, 9 were positive for two or more $\beta$-lactamase genes, whereas 5 isolates were negative. The study using the ampC kit identified 22 out of 30 previously characterized clinical isolates as positive for ampC $\beta$-lactamase genes. The remaining 8 isolates were correctly identified as negative samples. Therefore, the calculated clinical sensitivity and diagnostic specificity of both kits was determined to be $100 \%$. Representative amplification data of $\beta$-lactamase-positive clinical isolates using PCR Mix 3 of ARM-D Kit, $\beta$-Lactamase is shown in Figure 2.

\section{Conclusions:}

- Sensitivity and specificity were $100 \%$ for both kits.

- Each kit was tested on multiple instruments, including the Streck Zulu RT.

- Cycling times are approximately 45 minutes when tested on most real-time PCR platforms.

- Total cycling time is 22 minutes when using the Streck Zulu RT.

- Data demonstrate rapid multiplex real-time PCR detection of ESBLs, MBLs, KPCs, and plasmid-mediated ampC $\beta$-lactamase genes using Streck ARM-D Kits.

BioTechniques 61:212-213 (October 2016) doi 10.2144/000114464 